\title{
Probing Supersymmetric Baryogenesis: from Electric Dipole Moments to Neutrino Telescopes
}

\author{
Stefano Profumo \\ California Institute of Technology, MS 106-38 Pasadena, CA 91125
}

\begin{abstract}
We study the implications for electroweak baryogenesis (EWB) within the minimal supersymmetric Standard Model (MSSM) of present and future searches for the permanent electric dipole moment (EDM) of the electron, for neutralino dark matter, and for supersymmetric particles at high energy colliders. We show that there exist regions of the MSSM parameter space that are consistent with both present two-loop EDM limits and the relic density and that allow for successful EWB through resonant chargino and neutralino processes at the electroweak phase transition. We give present constraints on chargino/neutralino-induced EWB implied by the flux of energetic neutrinos from the Sun, the prospective constraints from future neutrino telescopes and ton-sized direct detection experiments, and the possible signatures at the LHC and at a future ILC.
\end{abstract}

Keywords: Supersymmetric Models, Baryogenesis, Electric Dipole Moments, Dark Matter PACS: $12.60 . J v, 98.80 . \mathrm{Cq}, 95.35 .+\mathrm{d}, 13.60 . \mathrm{Rj}$

As explained in detail in other Talks at this conference [1], EWB is a viable framework for the generation of a Baryon Asymmetry in the Universe (BAU) in the context of the MSSM. The crucial requirement of a sufficiently strongly first order EW phase transition forces however the choice of supersymmetric parameters into special and relatively strongly constrained regions of the MSSM parameter space. Specifically, the right-handed stop $\tilde{t}_{1}$ must be light (generically, lighter than the top quark), the heavier stop $\tilde{t}_{2}$ must be heavy (say, at the TeV scale), and, since CP violation in the SUSY is a necessary ingredient for EWB, sfermions must be heavy (for definiteness, in the present case-study we set $m_{\tilde{t}_{1}}=m_{t}$ and all the other sfermion masses to $10 \mathrm{TeV}$ ). The requirement of a sufficiently light Higgs mass forces the ratio of the up and down Higgs vacuum expectation values, $\tan \beta$, to be relatively small (we fix it to 10 here). Finally, as the BAU generated through EWB depends upon the heavy Higgs sector mass scale $m_{A}$, we fix the latter quantity to two representative values, 150 and $1000 \mathrm{GeV}$ (below $150 \mathrm{GeV}$ the SUSY contribution to the inclusive branching ratio $b \rightarrow s \gamma$ is too large, while for heavy $m_{A}>1 \mathrm{TeV}$ the BAU is too suppressed to be consistent with the observed value of the baryon-to-photon ratio [2]). In the framework of the above setting, the MSSM parameter space is reduced to four free parameters, entering the Neutralino (and Chargino) sector, namely the SU(2) and U(1) Gaugino masses $M_{2}$ and $M_{1}$, the SUSY Higgs mass parameter $\mu$, and the relative phase between the Higgsino and the Guagino mass parameters (the latter supposed to be proportional by virtue of some gauge-unification relation), $\phi_{\mu}[3]$.

Banning an extremely fine-tuned Stop sector, EWB in the MSSM gives rise to a sufficiently large BAU, compatibly with other phenomenological constraints, as long as $\mu \sim M_{2}$ (resonant Chargino baryogenesis) or $\mu \sim M_{1}$ (resonant Neutralino baryogenesis). The occurrence of this latter case, first pointed out in [3], opens up the possibility 


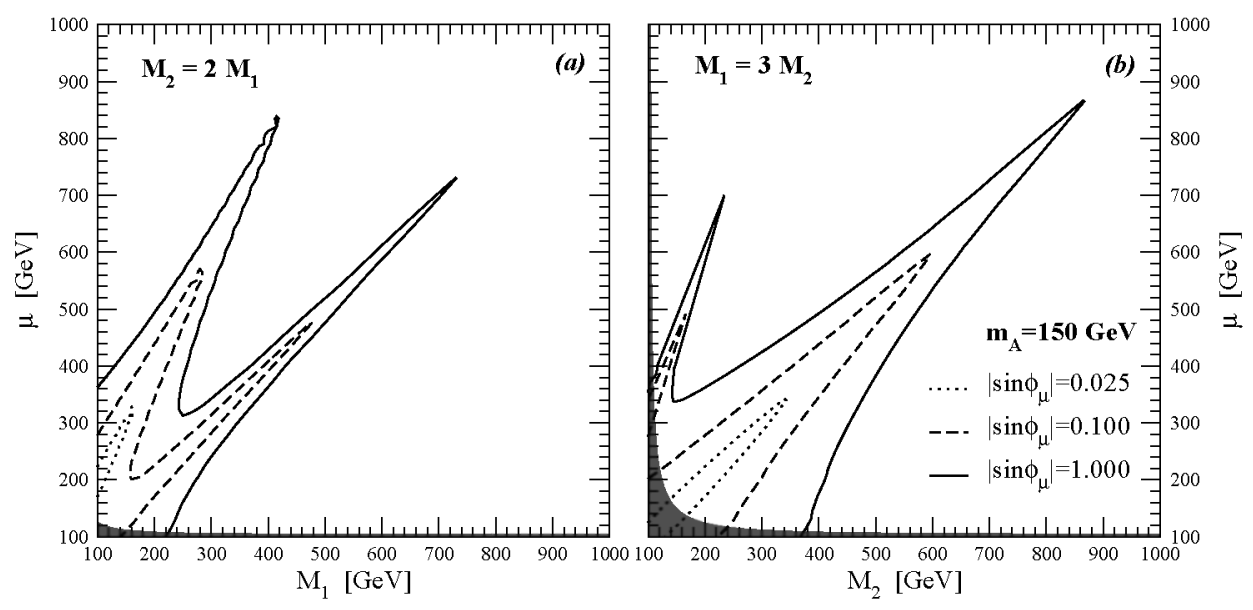

FIGURE 1. Regions, on the $\left(M_{1,2}, \mu\right)$ planes, producing the central WMAP value for the BAU [2], for various values of the CP-violating phase $\phi_{\mu}$, at $m_{A}=150 \mathrm{GeV}$. In panel $(a)$ we assume $M_{2} \simeq 2 M_{1}$, while in panel $(b)$ we take $M_{1} \simeq 3 M_{2}$. The regions shaded in grey correspond to chargino masses below the LEP2 limit, $\left.m_{\tilde{\chi}^{ \pm}}<103.5 \mathrm{GeV}\right)$.

that Dark Matter (DM), in the form of the stable lightest Neutralino, is connected to the mechanism for the generation of the BAU. In order to illustrate the portions of the MSSM parameter space where EWB is compatible with the observed BAU, we fix the ratio $M_{1} / M_{2} \approx 0.5$ (supergravity-like unification relation) and $M_{1} / M_{2} \approx 3$ (anomaly mediation relation). Fig. 1 illustrates, for fixed values of $\phi_{\mu}$ and at $m_{A}=150 \mathrm{GeV}$, the curves giving the central WMAP value for the BAU on the $\left(M_{1,2}, \mu\right)$ plane, and highlights three important facts: (1) the soft SUSY breaking parameters required by EWB and entering in the Neutralino and Chargino sector are generically well below the $\mathrm{TeV}$ scale; (2) the CP violating phase must be large enough ( $\left.\sin \phi_{\mu}>0.01\right)$; (3) the parameter space exhibits a two-funnel structure, corresponding to the Chargino and Neutralino driven resonant sources.

A very significant constraint on EWB in the MSSM is provided by the searches for electron, neutron or atomic electric dipole moments (EDM), as all those observables receive in general sizable contribution from SUSY radiative effects, as long as the SUSY particle masses are relatively light (say, below $\mathscr{O}(10 \mathrm{TeV})$ ), and the model contains SUSY sources of CP violation. In the present setup, the strongest constraint stems from the electron EDM, $d_{e}$. Even taking very large sfermion masses, a procedure that helps suppressing the one-loop charged Slepton-Neutralino and Sneutrino-Chargino loops, two loop contributions from loops of gauge and Higgs bosons and their SUSY partners are inevitable, and provide very serious constraints on the parameter space shown in Fig. 1. Ref. [3] showed, in fact, that maximal values for $\sin \phi_{\mu}=1$ are ruled out if one requires the right amount of BAU to be generated by EWB and one imposes the current experimental constraints on the size of $d_{e}$ [4]. This result applies even in the limit where the one-loop contributions are entirely neglected [3]. In Fig. 2 we suppose all sfermions are heavy, and therefore effectively neglect the aforementioned one-loop contributions, 

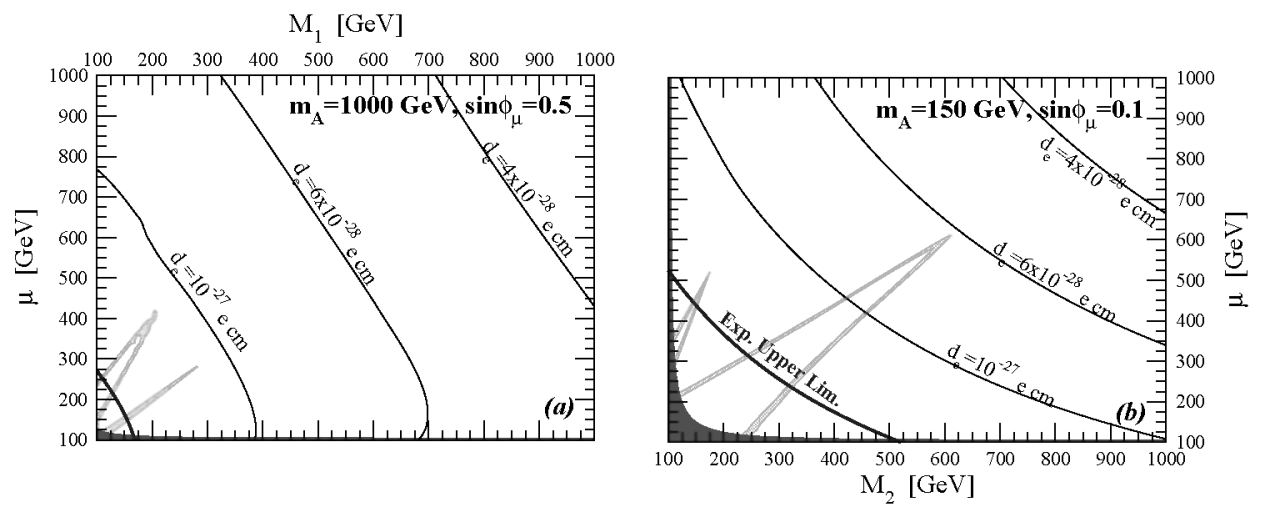

FIGURE 2. Iso-level curves of the electron electric dipole moment, on the $\left(M_{1,2}, \mu\right)$ planes, at $m_{A}=$ $1000 \mathrm{GeV}$ and $\phi_{\mu}=0.5$ (left) and at $m_{A}=150 \mathrm{GeV}$ and $\phi_{\mu}=0.1$ (right), in the limit of heavy sfermions. The thick dark grey lines represent the current experimental upper limit [4]. We also shade in light grey the 2- $\sigma$ regions corresponding to a WMAP BAU [2]. In the left panel we assume $M_{2} \simeq 2 M_{1}$, while in the right panel we take $M_{1} \simeq 3 M_{2}$.

and show curves at fixed values of $d_{e}$, as well as the current experimental upper limit [4]. The corridors compatible with the observed BAU are shaded in light grey. The value of $m_{A}$ is crucial to have at once successful EWB and consistency with the bounds on $d_{e}$, as both the EWB source terms and the two-loop EDM crucially depend upon the heavy Higgs sector mass scale. From the figure we deduce an important conclusion: EWB is compatible, for suitable choices of $m_{A}$ and $\sin \phi_{\mu}$, with current bounds from EDMs. Ref. [3] also pointed out that the requirement of successful EWB implies a lower bound on the expected size of $d_{e}>10^{-28} \mathrm{ecm}$. Remarkably enough, this value is well above

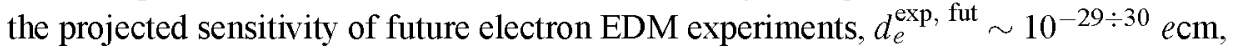
implying that EDM experiments will conclusively test the EWB scenario.

The quasi-degeneracy between $\mu$ and one of the gaugino masses plays a crucial role for DM detection, as long as $\mu$ is close to the smallest between the two gaugino masses (and, therefore, the lightest SUSY particle (LSP) features a sizable higgsino component). Not only would the LSP relic abundance be reduced (in the case of a bino-like LSP) or enhanced (for a wino-like LSP) by the occurrence of a sizable higgsino fraction, favoring the thermal production of neutralinos at a rate compatible with the cold DM abundance [2], but the coupling between the LSP and the lightest CP-even Higgs would also be largely enhanced. This latter feature plays a crucial role for the direct detection of DM $[8,9]$. As far as DM detection rates in the MSSM with CPV, Ref. [3] found that in the region of parameter space compatible with EWB a larger value of $\sin \phi_{\mu}$ leads to smaller direct detection rates (with respect to the $\mathrm{CP}$ conserving case) and larger fluxes of energetic neutrinos from neutralino pair annihilations in the core of the Sun. The left panel of Fig. 3 shows the region of the $\left(M_{1}, \mu\right)$ parameter space already constrained by direct detection [8] and by data on the flux of energetic neutrinos from the Sun [6]. Ref. [3] showed that the entire MSSM parameter space compatible with EWB will be probed by future ton-sized direct DM detection facilities [9], as well as by future $\mathrm{km}^{3}$ - 

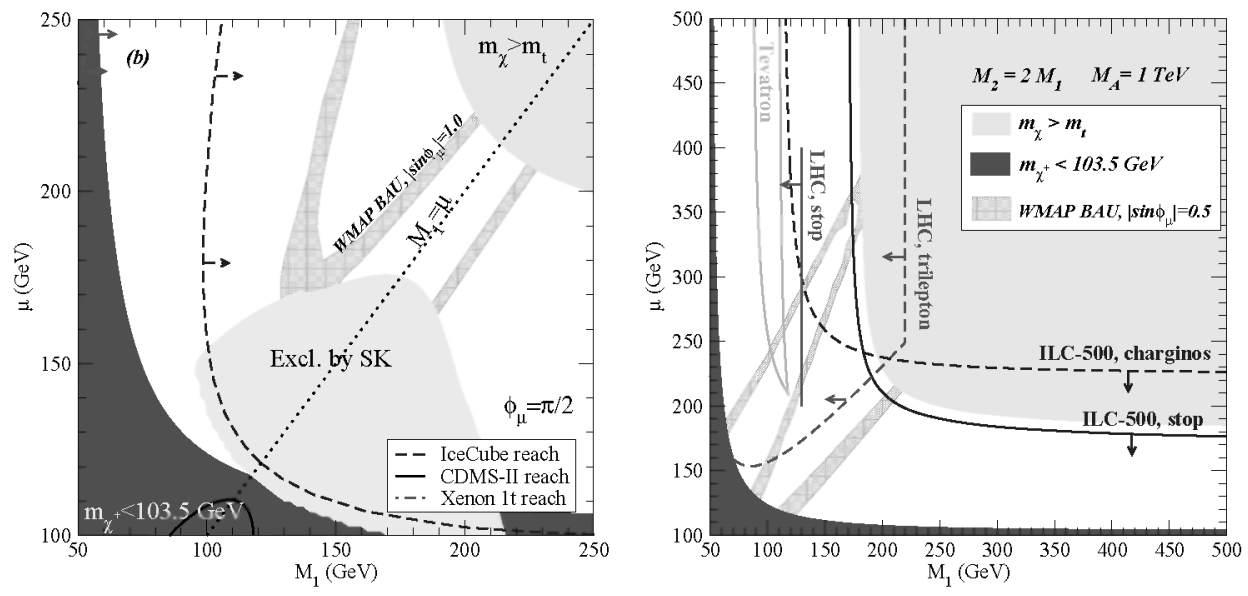

FIGURE 3. Left: Dark matter detection rates with maximal $\mathrm{CP}$ violation in the higgsino sector, on the $\left(M_{1}, \mu\right)$ plane, at $m_{A}=1 \mathrm{TeV}$. The central shaded region is already ruled out by the SuperKamiokande data on the flux of energetic neutrinos from the Sun [6], while the regions lying to the right of the blue dashed lines will produce a sizable flux of neutrinos at IceCube [7]. We also show the prospects for current [8] and future, ton-sized [9] direct detection experiments. The light blue shaded region corresponds to successful EWB; Right: A qualitative overview of next generation colliders reaches on the $\left(M_{1}, \mu\right)$ plane, at $m_{A}=1000 \mathrm{GeV}$ and $\sin \phi_{\mu}=0.5$. The color code for the shadings is the same as in the previous figures, and details on the sensitivity lines can be found in [3].

size neutrino telescaopes [7].

Finally, the right panel of Fig. 3 illustrates the reach of present and future colliders for the MSSM-EWB scenario: while the Tevatron reach will be very limited, the LHC can probe a sizable portion of the bino-like regions, while an ILC, with only $\sqrt{s}=0.5 \mathrm{TeV}$, will exhaustively explore all the viable parameter space of the model.

In conclusion, we showed that in many respects EWB in the MSSM gives rise to a falsifiable scneario, in the Popperian sense, as it can be simultaneously and unambiguosly probed by next generation (1) EDM experiments, (2) DM search experiments and (3) collider searches.

\section{REFERENCES}

1. See the talks and Proceedings by M. J. Ramsey-Musolf, C. Lee and S. Tulin at this Conference.

2. D. N. Spergel et al. [WMAP Collaboration], Astrophys. J. Suppl. 148 (2003) 175. See also

D. N. Spergel et al., arXiv:astro-ph/0603449 for the recent three year results.

3. V. Cirigliano, S. Profumo and M. J. Ramsey-Musolf, JHEP 0607 (2006) 002.

4. B. C. Regan, E. D. Commins, C. J. Schmidt and D. DeMille, Phys. Rev. Lett. 88 (2002) 071805.

5. D. Kawall, F. Bay, S. Bickman, Y. Jiang and D. DeMille, Phys. Rev. Lett. 92 (2004) 133007;

C. Y. Liu and S. K. Lamoreaux, Mod. Phys. Lett. A 19 (2004) 1235.

6. A. Habig, [Super-Kamiokande Collaboration], arXiv:hep-ex/0106024.

7. J. Ahrens et al. [IceCube], Nucl. Phys. Proc. Suppl. 118 (2003) 388.

8. D. S. Akerib et al. [CDMS Collaboration], arXiv:astro-ph/0509259.

9. E. Aprile et al., New Astron. Rev. 49 (2005) 289. 